\title{
Revisión del constructo lugar de control a partir de sus instrumentos de evaluación sobre poblaciones infantiles
}

\author{
Carmen Rodríguez-Naranjo, Antonio Godoy Avila, José \\ Antonio JimÉnez y Rosa Esteve Zarazaga \\ Universidad de Málaga
}

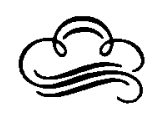

\section{Resumen}

Se realiza una revisión de las principales escalas de evaluación de las Expectativas de Lugar de Control existentes para niños y adolescentes, tanto en lo concemiente a sus características formales como a su fiabilidad y validez de constructo. El objetivo doble que se persigue reside, en primer lugar, en ofrecer una información que permita al lector escoger la escala más apropiada en función de los intere. ses que persiga. En segundo lugar, analizar el constructo a partir del cumplimiento de las predicciones realizadas por los autores de las mismas. Se discuten, al final, los ballazgos revisados en el contexto de la teoría del Aprendizaje Social y de la naturaleza y capacidad predictiva del constructo.

Palabras clave: Revisión, escalas de evaluación, lugar de control, expectativas, validez de constructo, fiabilidad.

\section{Abstract}

$A$ revision of the main Locus of Control expectancies assessment scales for children and teenagers is carried out concerning their formal charactesristics as well as their reliability and construct validity. There is a double aim in doing this, firstly, an information which allows the reader to choose the most appropriate scale according to their interests is given. Secondly, we analyze the construct from the fulfilment of the predictions made by their authors. Finally, we discuss the findings revised within the Social Leaming Theory context. We also discuss the nature and the predictive capacity of the construct.

Key words: review, assessment scales, locus of control, expectancies, construct validity, reliability.

Dirección de los autores: Departamento de Psicología de la Universidad de Málaga. 


\section{INTRODUCCION}

El constructo lugar de control deriva de la teoría del Aprendizaje Social de Rotter (Rotter, 1954, 1966; Rotter, Chance y Phares, 1972). Según esta teoría, la conducta humana tiene una direccionalidad u objetivos que se concretizan en la obtención de refuerzos. Por consiguiente, no deja de ser una teoría operante. Sin embargo, su novedad bien conocida y valorada reside en otorgar un papel destacado a una serie de variables cognitivas para la predicción de la conducta. En términos de la propia teoría, el «potencial de conducta» o probabilidad de que se produzca una conducta determinada en una situación dada dependerá fundamentalmente de las expectativas del reforzamiento, definidas como la creencia que tiene el sujeto de que tras una determinada conducta en una situación dada aparecerá un determinado refuerzo, del valor que para el sujeto tenga dicho reforzamiento, y de la situación psicológica.

La gran cantidad de estudios teóricos y empíricos que se han realizado en torno a las expectativas del refuerzo, variable denominada por Rotter (1966) «lugar de control», ha contribuido al logro de importantes hallazgos en torno a la predicción de la conducta. $Y$ ha supuesto una notoria confirmación de su utilidad explicativa. Sin embargo, en los estudios posteriores a la formulación original de la teoría, ha sido escaso el papel que se ha otorgado tanto al valor del refuerzo como a la situación psicológica.

El concepto lugar de control ha sido definido por Rotter (1966) como una expectativa generalizada sobre el origen de los refuerzos, estableciendo un continuo desde expectativas internas hasta expectativas externas. Postula como un principio general que «si el individuo interpreta un suceso reforzante como algo que depende de su propia conducta o de sus propias características relativamente constantes, se trata de una "expectativa de control interno". Cuando una persona percibe, en cambio, un suceso reforzante como algo que no depende del todo de su conducta y lo considera un resultado de la suerte, de la casualidad o del destino, se trata entonces de una "expectativa de control externo".» (Rotter, 1966, p. 1).

En su forma más simple, la hipótesis básica de Rotter (1966) supone que «si una persona percibe un reforzamiento como contingente a su propia conducta, entonces la ocurrencia de tal reforzamiento positivo o negativo fortalecerá o debilitará, respectivamente, el potencial para que dicha conducta vuelva a ocurrir en la misma o en otra situación similar. Si percibe el reforzamiento como fuera de su control o no contingente a su conducta (...), entonces es menos probable que la conducta precedente sea aumentada o disminuida» (Rotter, 1966, p. 5). A partir de esta asunción, han sido numerosos los estudios que han mostrado evidencia de que el aprendizaje bajo condiciones en las cuales la habilidad controla la consencuencia (o el sujeto cree que su habilidad la controla) es diferente del aprendizaje bajo condiciones en las cuales el sujeto cree que los resultados están determinados por la casualidad (Phares, 1957; Rotter, 1966; Rotter, Seeman y Liverant, 1962). En concreto, las condiciones de habilidad serían equivalentes a la expectativa de control interno, en tanto de las que casualidad se equipararían a la expectativa de control externo.

En esta línea, otros estudios experimentales muestran también que existen diferencias entre los sujetos con expectativas internas y externas en el tipo de tareas que prefieren. Así, los internos escogen tareas de habilidad frente a los externos, que, ante una situación de elección, se inclinan por tareas de azar (Ju- 
lian y Katz, 1968). Tal como han señalado Phares y Lamiell (1974) la preferencia por tareas de azar, en el caso de los externos, refleja una menor expectativa de éxito en tareas donde la habilidad controla la consecuencia, y viceversa en el caso de los internos. A partir de estos y otros hallazgos del mismo tipo, cabe inferir que las expectativas de lugar de control constituyen una variable que pude estar mediando, y por consiguiente puede explicar, las diferencias individuales en el aprendizaje que muestran los sujetos en situaciones donde las condiciones externas del mismo son similares.

La importancia teórica del lugar de control ha resultado evidente, por consiguiente, desde los primeros estudios realizados por los teóricos del aprendizaje social en la década de los cincuenta. Desde esta época han sido numerosas las investigaciones realizadas para evaluar la red nomológica de relaciones del concepto lugar de control con otras variables psicológicas: variables de identificación de los sujetos (edad, sexo y status socioeconómico, fundamentalmente), variables de rendimiento intelectual y académico, y otras variables comportamentales. Consecuentemente, han sido abundantes las escalas elaboradas para evaluar el constructo sobre sujetos pertenecientes a muestras de diferentes edades, conceptualizándolo como expectativas generalizadas o como expectativas específicas, como un constructo unidimensional o multidimensional, etc.

Debido al importante papel que los instrumentos de evaluación del constructo han jugado y siguen jugando para obtener información fiable y válida en torno a la naturaleza del mismo, en los siguientes apartados de este trabajo se revisan las características de las principales escalas de evaluación de las expectativas de lugar de control en poblaciones infantiles y de adolescentes, la relación que presentan unas con otras, su validez de contenido y estructura factorial. Se han elegido las escalas dirigidas a poblaciones infantiles por ser éstas (si exceptuamos la escala del propio Rotter) a partir de las que el constructo ha recibido una mayor y más fértil investigación.

Resumiendo, pues, con este trabajo se persigue un objetivo doble. Por un lado, realizar una presentación de los instrumentos más importantes de evaluación del lugar de control en poblaciones de niños y adolescentes, lo que aún no existe en castellano. Por otra parte, la revisión de la validez de contenido de estas escalas junto con el resto de propiedades psicométricas que se analizan tiene también la utilidad de servir como primer paso para realizar un análisis en torno a la naturaleza y propiedades del constructo.

\section{REVISION DE LOS PRINCIPALES INSTRUMENTOS DE MEDIDA PARA NIÑOS Y ADOLESCENTES}

En este apartado se va a hacer una revisión de las principales escalas diseñadas para evaluar las expectativas de lugar de control en niños y adolescentes. Se realiza una descripción de las características de cada uno de los instrumentos que se revisan en lo referente a su formato y a los presupuestos teóricos de los que parten sus autores para la elaboración de los mismos. También se indican, en caso de ser relevantes, los contextos de investigación que sirven de base para su construcción, especificando si evalúan el lugar de control a partir de la conceptualización original de Rotter o de otras diferentes.

Con estos objetivos, se divide la exposición en dos apartado: en el primero se recogen los datos indicados de los cinco instrumentos que han recibido una 
mayor cantidad de investigación hasta el momento, siendo éstos los más ampliamente utilizados con este tipo de poblaciones - la LCS de Bialer (1961), la IAR de Crandall y col. (1965), la CNS-IE de Nowicki y Strickland (1973), la SDRCI de Stephens y Delys (1973) y la Tel-Aviv de Milgram y Milgram (1975)_. En el segundo, se revisan menos exhaustivamente otras escalas que han recibido un menor número de investigaciones, pero que proporcionan una información útil sobre la capacidad predictiva del constructo.

\subsection{Instrumentos de medida de más amplia utilización}

a) La Bialer Locus of Control Scale (Bialer, 1961; LCS).

La LCS consta de 23 ítems, 18 con respuestas afirmativas y 5 con respuestas negativas, ambas puntuadas en la dirección interna. Se administra oralmente, de forma individual o en grupos, y va dirigida a niños de 6 a 15 años. Existe otra versión de la escala en la que se ha invertido la redacción de los ítems de tal forma que 18 respuestas negativas y 5 afirmativas puntúan en la dirección interna (Gozali y Bialer, 1968). Esta inversión en la redacción de los ítems ha sido llevada a cabo con el objeto de controlar la aquiescencia.

La LCS es la escala, de las que actualmente se utilizan para evaluar el lugar de control en poblaciones infantiles, que históricamente se propuso en primer lugar. Esta escala procede de la adaptación de un cuestionario de autoevaluación previo - diseñado para evaluar el constructo en poblaciones de adolescentes (James, 1957); revisado por Phares, 1957) -, y que ha sido sustituido posteriormente por la escala de Rotter (1966).

La LCS fue elaborada por su autor con el objeto de comprobar la suposición de Lewin (1935), según la cual sólo aquellos niños que pueden conceptualizar la relación entre su capacidad y el resultado de su conducta dirigida a una meta llegan a ser conscientes del éxito y el fracaso. Bialer (1961) considera que el niño va tomando conciencia, a lo largo del desarrollo, de su capacidad para influir en los resultados de los acontecimientos mediante sus propias acciones. Así, con el paso del tiempo y el aumento de su experiencia, crece la probabilidad de que vea los resultados de sus acciones dirigidas a una meta como una consecuencia de su propia conducta. De esta forma, el niño pasaría de considerar una gran cantidad de acontecimientos como buenos o malos a catalogarlos como éxitos o fracasos. Este cambio se hace posible conforme aumentan sus experiencias y el niño llega a considerar que muchas de las cosas que le suceden dependen de su conducta (expectativas internas). Por consiguiente, si sus experiencias son numerosas y variadas, cabe esperar que, con la edad, el niño pase de tener creencias en el control externo a tener creencias en el control interno, y desde la valoración de los sucesos como buenos o malos y de sus experiencias como agradables o desagradables, a su consideración como éxitos o fracasos. Desde esta perspectiva, en consecuencia, serán éxitos (o fracasos) las experiencias agradables (o desagradables) atribuidas internamente (al esfuerzo o a la capacidad, por ejemplo), pero no las atribuidas externamente (como al azar o a la suerte) (Bialer, 1961).

En suma, los tres cambios evolutivos que Bialer (1961) postula cabe esperar, y que están a la base de la construcción de su escala, son los siguientes: (a), un cambio desde las creencias de lugar de control externas a las creencias internas; (b), un cambio desde las primeras respuestas hedonistas a una sensibilización hacia las señales asociadas con el éxito y el fracaso; y (c), a partir del cambio 
de las expectativas externas a las internas, se espera también que se produzca una evolución de soportar mal la demora en la gratificación (en niños pequeños) a sobrellevarla bien, e incluso a preferir la gratificación demorada cuando el valor del refuerzo sea elevado.

b) El Intellectual Achievement Responsibility Questionnaire (Crandall, Katkovsky y Crandall, 1965; IAR).

Esta escala va dirigida a edades escolares a partir de los 4 años, aproximadamente. Se administra de forma oral a los más pequeños, y de forma escrita a los de los cursos superiores. Consta de 34 ítems con elección forzada entre dos alternativas, una interna y otra externa, repartiéndose por igual el número de ítems positivos y negativos.

Al igual que las escalas anteriores a su construcción, la IAR sigue la definición del lugar de control dada por Rotter (1966), proporcionando además tres puntuaciones: Internalidad positiva (I + ), Internalidad negativa (I-) e Internalidad total (It).

Crandall y col. (1965), en la creación de esta escala, parten de la consideración de que no existen razones para suponer que la creencia de lugar de control serán las mismas en los distintos campos situacionales, motivacionales o conductuales, por lo que las escalas debieran ir dirigidas a evaluar el constructo sobre distintas áreas. De hecho, desde la teoría del aprendizaje social no se postula que exista dicha generalidad transituacional y, además, tampoco se ha encontrado evidencia empírica de la misma. Debido a ello, los autores optan por elaborar una escala de naturaleza específica.

Por otra parte, ésta se desarrolla en un contexto de investigación relacionado con los comportamientos de logro y, en especial, con el desarrollo de la motivación de logro en ambientes académicos (Crandall y col., 1965). Ambas influencias sirven de marco para la elaboración de esta escala de lugar de control para niños, que se dirige a evaluar el constructo sobre situaciones de naturaleza intelectual-académica.

El objetivo de estos autores era averiguar qué variables podrían resultar de utilidad para predecir el comportamiento diferencial de los sujetos en situaciones de logro, ya que en este tipo de situaciones o tareas las dificultades para obtener el logro parecen estimular a algunos niños a un refuerzo mayor de persistencia en la tarea, en tanto que otros las abandonan con cierta rapidez. Postulan que, en este tipo de situaciones, las causas del esfuerzo para llegar a la meta se ubican fundamentalmente en las expectativas de control interno e inestable del refuerzo (Crandall y col; 1960).

Son todos estos aspectos los que influyen para que la escala de Crandall y col. (1965) se diferencie de las anteriores (James-Phares, 1957; Bialer, 1961) en los siguientes aspectos: (a), al quedar enfocada exclusivamente hacia las situaciones de logro intelectual-académico; (b), en las fuerzas ambientales externas descritas, que se limitan a aquellas personas que más a menudo toman contacto con el niño (padres, profesores e iguales), al ser éstos los que proporcionarán al niño los refuerzos de la conducta de logro descritos, la obtención de aprobación y la evitación del rechazo (no aparecen «otros poderosos», ni suerte, azar, etc.); y (c), al diferenciar entre resultados positivos y negativos; los autores piensan que la dinámica que opera para la obtención de buenos resultados $(\mathrm{I}+)$ puede ser muy diferente que la implicada para aceptar la resposabilidad de consencuencias desagradables (I-). 
c) La Cbildren's Nowicki-Strickland Locus of Control Scale (Nowicki y Strickland, 1973; CNS-IE).

La CNS-IE consta de 40 ítems que se contestan con «Sí» o «no». Las edades de aplicación van desde los 8 años hasta la adolescencia. Pero existen versiones especiales para niños de 4 a 8 años (PPNS-IE), y para estudiantes de "college» $\mathrm{y}$ adultos (ANS-IE).

Los autores no justifican teóricamente la necesidad de una nueva escala para la evaluación del constructo. Unicamente señalan que es necesario disponer de una medida metodológicamente válida que «pueda permitir a los investigadores describir mejor la red nomológica de relaciones que circundan esta dimensión» (Nowicky y Strickland, 1973, p. 149). De esta red de relaciones nomológicas destacan las referentes al desarrollo evolutivo de la internalidadexternalidad y a su asociación con el logro intelectual-académico, así como, a nivel discriminante, a ausencia de relación de la dimensión I-E con la deseabilidad social y con la inteligencia. Por consiguiente, para que ésta se pudiera considerar una medida adecuada del constructo, debe cumplir lo siguiente: (a), las puntuaciones deben aumentar en la dirección interna conforme se incremente la edad de los sujetos; (b), las puntuaciones deben relacionarse con el logro, obteniendo puntuaciones superiores en esta variable los internos que los externos; y (c), las puntuaciones de la escala no deben relacionarse significativamente con medidas de deseabilidad social o inteligencia.

Desde el punto de vista teórico, por tanto, la escala CNS-IE no hace aportación alguna con respecto a la LCS de Bialer (1961). No obstante, ha sido y continúa siendo una de las escalas infantiles más utilizadas, formando parte de una gran cantidad de investigaciones realizadas en torno a la validez factorial y predictiva del constructo.

d) La Stephens-Delys Reinforcement Contingency Interview (Stephens y Delys, 1973; SDRCI).

Esta escala, dirigida a niños de edad preescolar, consta de 40 ítems de respuesta libre, la mitad positivos y la otra mitad negativos. Cada pregunta plantea la ocurrencia de un esfuerzo social y pide al niño que proporcione la conducta sobre la que ese refuerzo sería contingente; por ejemplo, «Qué hace sonreír a las madres?». Por consiguiente, es de administración oral e individual. Esta es la primera escala dirigida expresamente a niños de edad preescolar, concretamente de 3 y 4 años.

Stephens y Delys (1973) piensan que las expectativas externas son las responsables principales del bajo logro académico de algunos niños, que a su vez los confirman en sus expectativas externas, las que a su vez revierten en que dicho bajo logro se siga manteniendo en una especie de círculo vicioso.

Los refuerzos que se representan en su escala, sin embargo, son todos alusivos a alguna «consecuencia social» (sonreír, hacer feliz, enfadarse, etc.) y ninguno de ellos hace referencia a las consecuencias más directas del comportamiento académico (aprobar, suspender, etc.), quizás a causa de la poca edad de los niños a los que va dirigida la prueba. Respecto a los agentes de refuerzo considerados, éstos son las madres, los padres, los profesores, otros niños y el propio niño que responde a la escala.

e) La Tel Aviv Locus of Control Scale (Milgram y Milgram, 1975; TA).

Esta prueba consta de dos escalas de 24 ítems cada una: la Escala de Pasado y la Escala de Futuro. La mitad de los ítems de cada escala se refieren a resulta- 
dos de éxito y la otra mitad a resultados de fracaso, con dos subescalas cada una de 12 ítems positivos y otros 12 negativos. Cada ítem consiste en una sentencia en la que se describe la ocurrencia de un refuerzo y de varias opciones de respuesta entre las que el sujeto debe elegir una. Las edades de aplicación van desde los 6 años, aproximadamente, en adelante. La administración se lleva a cabo en grupo.

Milgram y Milgram (1975) desarrollaron esta prueba en el supuesto de que el lugar de control debe considerarse como un constructo multidimensional, tal como señalaban los estudios previos sobre la escala I-E de Rotter. En este sentido, los Milgram concuerdan con las asunciones de Crandall y sus colaboradores (1965) al distinguir entre resultados positivos y negativos, así como en la necesidad de atender a la posible especificidad de situaciones, conductas y refuerzos a través de los cuales se generalizan las expectativas de lugar de control (situaciones a las que se denomina «loci»). En lo referente a las situaciones representadas en los «loci», la prueba consta de ítems tomados de tres importantes enclaves de la vida del niño: la escuela (relación con el maestro y con el currículum), el hogar (relación con padres y hermanos) y la vecindad (relación con los iguales en edad, tanto familiares como no familiares).

Por otra parte, Milgram (1972), utilizando la LCS de Bialer, había encontrado en una muestra de deficientes que, en general, la puntuación de internalidad que se obtenía en los ítems que hacían referencia al pasado era mayor que la obtenida en los ítems con referencia al futuro. Además, se encontraba en este tipo de población que en tanto que los sujetos se atribuían a sí mismos el éxito y se culpaban por el fracaso en los resultados pasados, no expresaban confianza en su habilidad para influenciar los resultados futuros. Esto llevó a los Milgram a añadir una dimensión más, relacionada con el tiempo (pasado/futuro).

En lo concerniente a los factores atribucionales representados en la escala, éstos son, del lado interno, el esfuerzo, y del lado externo, fuerzas externas incontrolables. Los autores postulan - siguiendo las relaciones propuestas por Joe (1971) entre puntuaciones internas y conductas constructivas - que cuando los sujetos responden en una dirección interna, se pueden hacer las siguientes suposiciones: (a), el sujeto cree que sus esfuerzos pueden influir en los resultados futuros; (b), el sujeto que responde en esta dirección realiza mayores esfuerzos que el que atribuye en la dirección externa; y (c), los esfuerzos que realiza el sujeto que responde en una dirección interna dan como resultado conductas más constructivas.

Dado que al postular validez predictiva para las puntuaciones de la Escala de Pasado es necesario suponer que el sujeto generaliza sus atribuciones sobre la experiencia pasada a las expectativas sobre lo que ocurrirá en el futuro, en tanto que en la Escala de Futuro no, Milgram y Milgram (1975) postularon que la relación entre esta última y otras variables comportamentales sería mayor que la existente entre dichas variables y las puntuaciones de la Escala de Pasado. Los datos empíricos, sin embargo, mostraron una relación no despreciable entre las escalas de Pasado y de Futuro, especialmente en los niños más mayores (a partir, aproximadamente, de los 7 años), lo que, unido a la ausencia de validez predictiva diferencial de esta dimensión, ha llevado a que algunos autores pongan en duda la utilidad de la distinción entre ítems referidos al pasado y referidos al futuro (Gilmor, 1978). 


\section{8}

\subsection{Otras escalas de evaluación del constructo en poblaciones infantiles}

Además de las escalas anteriormente comentadas existen otras muchas, tanto para evaluar las expectativas generalizadas como las más específicas. A continuación se describen brevemente algunas de las que son más utilizadas.

a) La Children's Picture Test of Internal-External Control (Battle y Rotter, 1963).

Esta escala consta de ítems acompañados de una tarjeta cada uno en la que hay dibujada una escena de interacción. Se administra de forma individual, ya que se pide al niño que describa lo que diría si se encontrara en la situación que representa el dibujo. Para calcular las puntuaciones que los niños obtienen en el Battle-Rotter, una serie de jueces entrenados deben valorar las respuestas de éstos en una escala de siete puntos, según consideren que expresan expectativas internas $(1,2$ y 3$)$, externas $(5,6$ y 7$)$ o que no es posible decidir con seguri$\operatorname{dad}(4)$.

Battle y Rotter (1963) presentaron su escala en el contexto de un estudio que se proponía explorar la interacción de la clase social y el grupo étnico con el lugar de control. La hipótesis principal de los autores plantea que los sujetos que pertenezcan a clases socioeconómicas superiores estarán menos controlados por fuerzas externas que los socialmente desfavorecidos y, por consiguiente, tendrán una actitud de mayor dominio sobre el ambiente. Se apoyan en el estudio de Seeman (1963), en el cual, analizando el efecto que la alienación producía en el aprendizaje sobre una muestra de sujetos sometidos a prisión, encontró que los presos alienados aprendían significativamente menos material relevante para obtener la libertad que los menos alienados. No obstante, hay que indicar que este estudio hace referencia al control objetivo que tienen los sujetos, lo que es diferente de las expectativas de lugar de control.

Battle y Rotter (1963) consideran, por consiguiente, que cabe esperar una relación significativa de las puntuaciones internas de su escala con las siguientes variables: (a), el status socioeconómico, obteniendo puntuaciones más externas las clases más desfavorecidas; y (b), la ejecución de los niños en situaciones de logro, a partir de los hallazgos obtenidos previamente sobre la escala de Bialer (1961).

b) La Standford Preschool Internal-External Control Scale (Mischel, Zeiss y Zeiss, 1974; SPIES).

Esta escala consta de 14 ítems de elección forzada entre una alternativa interna y otra externa. La mitad de los ítems describen eventos positivos y la otra mitad, negativos. Es de administración oral e individual, con edades de aplicación que van desde los 3 a los 6 años, aproximadamente.

Mischel, Zeiss y Zeiss (1974) parten del constructo amplio de expectativas generalizadas del lugar de control de Rotter (1966), llegando a considerar que tales expectativas generalizadas probablemente funcionen de una forma muy parecida a los rasgos que postulan las teorías de personalidad tradicionales.

Los aspectos que definen las aportaciones de esta nueva escala para evaluar el lugar de control en poblaciones de edad preescolar pueden definirse por: (a), utilizar un formato de ítems de elección forzada para evitar la tendencia de los niños de estas edades a contestar afirmativamente en respuestas de sí-no; y (b), la utilización de las subescalas positiva y negativa.

c) La Preschool and Primary Nowicki-Strickland Internal-External Control Scale (Nowicki y Duke, 1974; PPNS-IE). 
Esta escala es muy semejante a la CNS-IE de Nowicki y Strickland (1973), ya que de hecho supone una adaptación de sus ítems para niños de edad preescolar. Se constituye, por consiguiente, como una medida generalizada del constructo, al igual que la anterior.

Esta escala es de administración grupal, dirigida a niños de 4 a 8 años. Utiliza un formato de respuesta sí-no, mostrándose cada ítem en una cartulina en la que se presenta la interacción de dos personajes masculinos (PPNS-IE para varones) o la interacción de dos personajes femeninos (PPNS-IE para niñas).

Respecto a las relaciones esperadas de las puntuaciones de la escala con otras variables, éstas son las mismas que las postuladas para la CNS-IE. De hecho, no supone más que una adaptación de la anterior.

d) La Gruen-Korte-Stephens Internal-External Scale (Gruen, Korte y Baum, 1974).

La escala consta de 38 ítems ilustrados cada uno con personajes recortados y pegados, administrándose las instrucciones mediante una grabación magnetofónica. Las edades de aplicación comprenden de los 3 a los 8 años, aproximadamente. Sin embargo, entre los 6 y 8 años la escala discrimina muy mal entre los sujetos internos y externos.

Esta escala ha sido elaborada por sus autores con el objeto de crear un instrumento de evaluación que minimice el efecto del sesgo cultural y socioeconómico sobre las puntuaciones de lugar de control. Para conseguir este objetivo, se crearon ítems que requirieran una capacidad verbal mínima para comprender su contenido.

e) La Academic Achievement Accountability (Clifford y Cleary, 1972; revisión de Clifford en 1976; AAA).

Esta escala consta de 18 ítems que puntúan en la dirección interna, con 5 opciones de respuesta cada uno con el objetivo de evaluar la magnitud de las respuestas. La edad de aplicación es de 4 a 9 años, aproximadametne, pudiéndose realizar individualmente o en grupos.

Sus autores construyeron esta escala con el objeto de evaluar la dimensión I-E en contextos académicos. Por consiguiente, es semejante a la IAR de Crandall y col. (1965). Sin embargo, estas dos escalas se diferencian en lo siguiente: (a), el polo externo de la IAR hace referencia a los otros significativos (padres, profesores, amigos), y a la dificultad de la tarea, en tanto que la AAA lo refiere a fuerzas externas incontrolables (azar, suerte, destino); y (b), la IAR incluye, en el polo interno, la capacidad como factor explicativo, en tanto que la AAA no lo hace.

Puede decirse, por tanto, que esta escala evalúa el locus interno-externo en el polo inestable de la dimensión estabilidad-inestabilidad (según la nomenclatura de Weiner). El componente estable (capacidad y dificultad de la tarea) se intenta mantener constante al hacer referencia a lo que «suele ser usual en la vida del sujeto» (v. gr., «Cuando actúas peor de lo normal...»).

f) La Locus of Control Scale for Children's Perceptions of Social Interactions (Dahlquist y Ottinger, 1983; LOC-CPSI).

Esta escala consta de 40 ítems con formato de respuesta sí-no, 20 de ellos indicando internalidad y 20 externalidad, tanto sobre resultados positivos como negativos. Va dirigido a niños de 4 a 7 años, aproximadamente.

Esta es la primera prueba dirigida a evaluar las creencias de lugar de control 
en situaciones de interacción social sobre niños de tan corta edad. Esto hace que las categorías de interacción social muestreadas sean las siguientes: conductas de interacción en clase, en el recreo y con los amigos de la vecindad. La dimensión de lugar de control evaluada es únicamente el continuo interno-externo tal como fue definido por Rotter (1966), para lo cual se tuvieron en cuenta las valoraciones hechas por jueces.

\subsection{Escalas para la evaluación de las expectativas de lugar de control en niños y adolescentes elaboradas en nuestro medio}

a) El Cuestionario LUCAD (Pelechano y Báguena, 1983).

El LUCAD consta de 88 sentencias que se responden mediante las opciones «verdadero» y «falso». Su edad de aplicación corresponde al tercer ciclo de la EGB.

Esta escala ha sido diseñada en nuestro medio siguiendo el ejemplo de varias escalas extranjeras. Los análisis sobre la bondad psicométrica de la misma han sido realizados a partir de una muestra de 1.036 alumnos de centros en su mayoría públicos, una segunda muestra de 911 alumnos de escuelas rurales y una tercera muestra de 198 alumnos y 235 alumnas de centros estatales, todas pertenecientes al tercer ciclo de la EGB.

El análisis factorial realizado sobre este cuestionario indica la presencia de dos factores externos y otros dos internos: «Control externo en las relaciones sociales», «Control externo: exculpación y atribución a factores físicos, sociales y al paso del tiempo», «Control interno en logros» y «Control interno: voluntariedad-responsabilidad y acción directa». Dado que esta estructura factorial es más simple que la encontrada por Pelechano y Báguena para el LUCAM (la escala equivalente para adultos), los autores concluyen que sus resultados confirman la evolución psicológica de las expectativas de lugar de control hacia un progresivo enriquecimiento y complejidad.

Respecto a la consistencia interna de la prueba, los coeficientes omega calculados para cada uno de los cuatro factores anteriores son satisfactorios, oscilando de 0,45 a 0,72 .

Las relaciones calculadas entre las puntuaciones de la prueba y el PMA multifactorial de inteligencia de Thurstone son en su mayoría estadísticamente significativas, presentando una asociación superior con los factores verbales referidos al uso significativo del vocabulario y procesos complejos que los no verbales o la afluencia verbal simple. Por su parte, las relaciones con el rendimiento académico resultan negativas excepto para el factor «Control interno: voluntariedadresponsabilidad y actuación directa».

Por último, mediante otros análisis se muestra cómo el cuestionario LUCAD está conformado por factores que atienden a aspectos psicológicos nuevos no muestreados por otro tipo de cuestionarios existentes previamente en castellano.

b) Las escalas ELC-A y ELC-I (Godoy, Rodríguez-Naranjo, Esteve y Silva, 1989).

Estas escalas van dirigidas a evaluar las dimensiones de internalidadexternalidad y controlabilidad-incontrolabilidad, tanto en situaciones académicas (la ELC-A), como en situaciones interpersonales (la ELC-I), evaluando resultados positivos y negativos. También ofrece una puntuación general (ELC-G).

La ELC-A consta de 34 ítems, y la ELC-I, de 32. Cada ítem está formado 


\section{1}

por una afirmación y cuatro opciones de respuesta, desde mayor a menor internalidad o, en su caso, desde mayor a menor controlabilidad. Cada una de las escalas proporciona cuatro tipos de puntuaciones: Internalidad-externalidad para resultados positivos (IE + ), Internalidad-externalidad para resultados negativos (IE-), Controlabilidad-incontrolabilidad para resultados positivos $(\mathrm{CNC}+$ ) y Controlabilidad-incontrolabilidad para resultados negativos (CNC-). La edad de aplicación va desde los 8 hasta los 18 años.

Para la elaboración de las escalas, se ha tenido en cuenta una serie de supuestos teóricos que se consideran pertinentes para la evaluación del constructo a partir de los estudios teóricos realizados y los hallazgos obtenidos sobre escalas anteriores. Estos son los siguientes: (a), la importancia de la valencia de los resultados, a partir de los datos empíricos proporcionados por algunos autores (Crandall y col., 1965; Gregory, 1978; Milgram y Milgram, 1975; Mischel y col., 1974; etc.); (b), la ausencia de valor teórico y empírico en la diferenciación entre causas atribuidas sobre resultados pasados y causas atribuidas sobre resultados futuros (Clifford, 1972; Clifford y Cleary, 1972; Crandall y col., 1965; Mischel y col., 1974; Rotter, 1966); (c), la no diferenciación entre atribuciones y expectativas al no aportar ésta capacidad predictiva sobre conductas y/o refuerzos (Brewin y Shapiro), 1984); (d), la diferenciación entre las dimensiones de internalidad-externalidad y controlabilidad-incontrolabilidad, fundamentalmente a partir de la especificidad predictiva de cada una de ellas (Weiner, 1979); y (e), la especificidad de las expectativas de lugar de control para diferentes áreas de contenido (Crandall, Katkovsky y Crandall, 1965; Rotter, 1975).

Partiendo de estos supuestos, los autores elaboran los ítems que componen estas escalas a partir de un muestreo de situaciones, comportamientos y refuerzos pertinentes para los niños de estas edades, llevado a cabo tanto por la revisión de las escalas infantiles anteriores como por la realización de un estudio piloto elaborado a este fin.

La bondad psicométrica de la escala ha sido calculada sobre 5 muestras de EGB, constituyendo un total de 540 sujetos de los cursos comprendidos entre $4 .^{\circ}$ de EGB y $3 .^{\circ}$ de BUP. Respecto a los datos de fiabilidad, los coeficientes alfa de Cronbach de cada dimensión para cada resultado (IE +, IE-, CNC +, $\mathrm{CNC}-$ ), en ambas escalas, fluctúan entre 0,70 y 0,85 , lo que sus autores consideran adecuado sobre todo si se atiende a la diversidad de comportamientos y resultados a los que hacen referencia los ítems.

Por otra parte, y ya en lo que respecta a la validez de la escala, la relación entre las dimensiones IE y CNC es o bien nula o bien moderada, lo que es tomado como una prueba empírica de la distinción entre ambas dimensiones.

$\mathrm{Al}$ estudiar la composición factorial de las escalas, Godoy y colaboradores han encontrado que los ítems se agrupan, como se esperaba, en torno a cuatro factores perfectamente coincidentes con las dimensiones internalidad-externalidad y controlabilidad-incontrolabilidad, para resultados positivos y negativos.

A partir de los estudios realizados, estas escalas no presentan relaciones estables con la edad, excepto en la dimensión de controlabilidad para resultados positivos. Y respecto al nivel socioeconómico de los sujetos, las relaciones son también escasas. Para explicar ambos resultados, los autores atienden a los argumentos aducidos por Skinner y Chapman (1987) acerca del diferente curso evolutivo de los distintos factores atribucionales, a partir de lo que cabe esperar que los resultados fluctúen en función de los factores muestreados por cada escala.

Por otra parte, las relaciones encontradas con la inteligencia son bastante 
ilustrativas respecto al valor predictivo de las dimensiones propuestas; así, en tanto que no se encuentran relaciones con la ELC-A, lo que entienden los autores que aporta validez discriminante a la escala, sí aparecen algunas relaciones ligeras para la ELC-I, indicando que cuanto mayor es la inteligencia más se atribuyen los éxitos y fracasos a factores internos. A su vez, cuanto mayor es la inteligencia, tanto más controlables se ven los éxitos, pero no así los fracasos.

Igualmente coherentes con la teoría de Weiner (1980) son las relaciones con las calificaciones escolares en el caso de la ELC-A, la ejecución en tareas cognoscitivas y el autoconcepto. En concordancia con las predicciones realizadas, y al igual que ocurre en el caso citado de la inteligencia, los autores encuentran diferentes patrones de asociación con estas variables en función del tipo de situación (ELC-A o ELC-I), la dimensión evaluada (internalidad-extẹnalidad o controlabilidad-incontrolabilidad) y el tipo de resultado (positivo o negativo).

\section{FIABILIDAD DE LAS PRINCIPALES ESCALAS DE LUGAR DE CONTROL PARA NIÑOS Y ADOLESCENTES}

Los coeficientes de fiabilidad de las escalas de evaluación del lugar de control hacen referencia fundamentalmente a la consistencia interna y a la estabilidad temporal. A continuación se revisan los coeficientes obtenidos en las principales escalas.

\subsection{Concondancia entre jueces}

Unicamente se ha utilizado este procedimiento para calcular la fiabilidad de las escalas de lugar de control en el caso de la Children's Test of I-E Control (Battle y Rotter, 1963), y en la Stephens-Delys Reinforcement Contingency (Stephens y Delys, 1973) a causa de la naturaleza abierta de sus respuestas. El coeficiente de correlación de Pearson que expresa la concordancia entre los jueces utilizados por los autores, sobre una muestra de 40 protocolos fue de 0,93 , en la escala de Battle y Rotter; y de 0,98 , en la Stephens-Delys.

Respecto a la primera, este índice de la adecuación del procedimiento de puntuación de la escala es lo único de que se dispone como medida de su fiabilidad. Conviene indicar, igualmente para ambos casos, que el coeficiente de correlación de Pearson resulta inadecuado en aquellas ocasiones en las que se utilizan más de dos jueces - lo que de hecho se dio en los estudios de Battle y Rotter y de Stephens y Delys-. En ambos, por tanto, parece que hubiera resultado más apropiado haber utilizado el coeficiente W de Kendall.

\subsection{Formas paralelas}

La única escala que posee forma paralela es la LCS de Bialer. Esta forma paralela, creada por Gozali y Bialer (1968), se hizo invirtiendo la redacción de los ítems de la escala original (Bialer, 1961). La correlación entre las respuestas según ambos formatos es de 0,67.

\subsection{Consistencia interna}

En el Cuadro 1 se presentan los coeficientes de consistencia interna hallados por diferentes estudios para las principales escalas de lugar de control in- 


\section{3}

fantiles y de adolescentes. Se incluye el tipo de muestra y el número de sujetos que la componen en aquellos casos en los que la literatura hace estos datos disponibles.

\section{Cuadro I}

\begin{tabular}{|c|c|c|c|c|c|}
\hline \multirow[t]{2}{*}{ Formas paralelas } & \multicolumn{4}{|c|}{ Por mitades } & $N^{\circ}$. total \\
\hline & $\begin{array}{l}\text { Muestra } \\
\text { normal }\end{array}$ & \multicolumn{3}{|c|}{$\begin{array}{l}\text { Muestra } \\
\text { retrasados }\end{array}$} & \\
\hline LCS (Bialer, 1961) 0,67 & 0,49 & \multicolumn{3}{|c|}{0,87} & 36 \\
\hline AAA (Clifford y Cleary, 1972) & \multicolumn{4}{|c|}{0,66 y 0,67} & \\
\hline \multirow{3}{*}{ IAR (Crandall y col., 1965) } & $\begin{array}{l}\text { M. cursos } \\
\text { inferiores }\end{array}$ & \multicolumn{3}{|c|}{$\begin{array}{l}\text { M. cursos } \\
\text { superiores }\end{array}$} & \multirow{3}{*}{260} \\
\hline & It $\mathrm{I}+\mathrm{I}-$ & It & I + & $\mathrm{I}-$ & \\
\hline & $0,54 \quad 0,57$ & & 0,60 & 0,60 & \\
\hline & It & $\mathbf{I}+$ & & I- & \\
\hline CPSI (Dahlquist y Ottinger, 1983) & 0,83 & 0,76 & & 0,80 & \\
\hline \multirow[t]{2}{*}{ Gruen y col. (1974) } & \multicolumn{4}{|c|}{0,76} & \\
\hline & It & $I+$ & & I- & \\
\hline $\begin{array}{l}\text { SPIES (Mischel y col., 1974) } \\
\text { (edad preescolar) }\end{array}$ & No signif. & 0,14 & & 0,20 & 211 \\
\hline
\end{tabular}

Cada item con el total

PPNS-IE (Nowicki y Duke, 1974)

De 0,20 a 0,30

(edad preescolar)

$\frac{240}{\text { De } 0,20 \text { a } 0,30}$

\begin{tabular}{lccc}
\hline & $\begin{array}{c}\text { M. cursos } \\
\text { inferiores }\end{array}$ & $\begin{array}{c}\text { M. cursos } \\
\text { superiores }\end{array}$ & \\
\cline { 2 - 3 } & 0,60 & 0,81 & 1.017 \\
$\begin{array}{l}\text { CNS-IE (Nowicki } \\
\text { y Strickland, 1973) }\end{array}$ & 0,61 & \\
\hline
\end{tabular}

Cada item con el total

SDRCI (Stephens y Delys, 1973) (edad preescolar)

\begin{tabular}{lccc}
\hline & \multicolumn{1}{c}{$\begin{array}{c}\text { Subescala } \\
\text { pasado }\end{array}$} & $\begin{array}{c}\text { Subescala } \\
\text { futuro }\end{array}$ & \\
\cline { 2 - 3 } & De 0,31 a 0,67 & De 0,74 a 0,93 & 298 \\
$\begin{array}{l}\text { TEL AVIV (Milgram } \\
\text { y Milgram, 1975) }\end{array}$ & & & \\
\hline
\end{tabular}

(Original de Rodríguez-Naranjo et al., 1990) 
Tal como puede verse en este cuadro, la consistencia interna de muchas de las escalas de lugar de control es más bien moderada. Así ocurre, en el caso de las escalas generalizadas, por ejemplo, tanto en la LCS de Bialer (1961), Miller (1960), Shaffer y col. (1969) como en la IAR de Crandall y col. (1965).

Aún más bajos (de 0,14 a 0,30 ) se presentan los coeficientes tanto para la PPNS-IE como la SPIES, ambas escalas dirigidas a niños de edad preescolar. Respecto al primer caso, un estudio de Herzberger y col. (1978) indica también que, considerados aisladamente los ítems de esta escala, ninguno de ellos correlaciona positivamente con la puntuación total obtenida en la mayoría de los subgrupos de su estudio. No ocurre así, sin embargo, en el caso de la SDRCI, cuyos índices alcanzan coeficientes de correlación de 0,91 (Stephens, 1971, 1972).

Por otra parte, los coeficientes de consistencia interna calculados sobre la IAR y la CNS-IE muestran que éstos ascienden conforme se incrementa la edad de los sujetos de la muestra, pudiéndose analizar en el mismo sentido los bajos coeficientes obtenidos con la SPIES y la PPNS-IE, ambas dirigidas a niños en edad preescolar.

En esta línea, son interesantes los hallazgos de Gorsuch y col. (1972) sobre la LCS de Bialer, que indican que la capacidad verbal de los sujetos y la consistencia interna de la escala presentan una relación lineal. Los coeficientes se incrementaban de forma gradual, desde 0,10 para el grupo de capacidad más baja hasta 0,60 para el de capacidad más alta. Este mismo patrón de resultados ha sido confirmado sobre la CNS-IE, mostrando una relación significativa entre capacidad verbal y los índices de consistencia interna de esta escala sobre algunos grupos de edad (Halpin y Otinger, 1983).

Una gran cantidad de estudios indican coeficientes de consistencia interna generalmente superiores en el caso de la CNS-IE que los calculados sobre otras escalas generalizadas (Halpin y Ottinger, 1983; Nowicky y Strickland, 1973; Tesiny, Lefcowitz y Gordon, 1980). La otra escala de lugar de control generalizado que también ofrece coeficientes similares, de 0,76 , aunque no se utilizan para el cálculo de su consistencia interna muestras de diferentes edades, es la de Gruen y col. (1974).

Respecto a la otra escala que junto con la IAR va dirigida a evaluar el constructo sobre el área académica, la AAA, ofrece coeficientes similares a la anterior en su forma original, y superiores en la revisada por Clifford (1976). Sin embargo, la otra escala de naturaleza específica y que también distingue entre $\mathrm{I}+$ e I-, la CPSI dirigida a situaciones de interacción social, ofrece índices superiores (Dahlquist y Ottinger, 1983).

Por último, señalar cómo también se presentan bastante altos los coeficientes de consistencia interna en el caso de la escala de Futuro de la Tel Aviv, en tanto que los correspondientes a la escala de Pasado son muy inferiores (Milgram y Milgram, 1975).

De cualquier manera, debido en parte a la señalada influencia de la capacidad verbal, y en mayor medida a la frecuente evaluación del lugar de control como un constructo de expectativas generalizadas, en ningún caso se suele considerar que la baja consistencia interna suponga un problema para la evaluación del mismo. Sin embargo, sí debiera esperarse que cuanto más específicas sean las escalas en torno a las situaciones, las conductas y los refuerzos evaluados, presentarán una mayor consistencia interna, lo cual no queda confirmado en escalas de este tipo, como es el caso de la IAR de Crandall y col. (1965), que ofrece coeficientes moderados. 


\subsection{Estabilidad temporal}

Tal como puede verse en el Cuadro 2, la estabilidad temporal o fiabilidad test-retest de las escalas de evaluación del lugar de control suele arrojar índices superiores a los de consistencia interna.

\section{CuAdro II}

Estabilidad temporal de las escalas de lugar de control para niños y adolescentes

\begin{tabular}{lllll}
\hline LCS (Bialer, 1961) & \multicolumn{3}{c}{0,84} & $\mathrm{~N}^{0}$ tot. \\
\hline & It & I+ & I- & \\
\cline { 2 - 4 } IAR (Crandall y col., 1965) & 0,69 & 0,66 & 0,74 & 130 \\
\hline
\end{tabular}

CPSI (Dahlquist y Ottinger, 1983)

De 0,68 a 0,70

Gruen y col. (1974) 0,83

\begin{tabular}{|c|c|c|c|}
\hline & Varones & Niñas & \\
\hline $\begin{array}{l}\text { SPIES (Mischel y col., 1974) } \\
\text { (edad preescolar) }\end{array}$ & De 0,55 a 0,70 & De no signif. a 0,65 & 211 \\
\hline $\begin{array}{l}\text { PPNS-IE (Nowicki y Duke, 1974) } \\
\text { (edad preescolar) }\end{array}$ & \multicolumn{2}{|c|}{0,79} & 60 \\
\hline $\begin{array}{l}\text { CNS-IE (Nowicki y Strickland, } \\
\text { 1973) }\end{array}$ & \multicolumn{2}{|c|}{ De 0,63 a 0,71} & 1.017 \\
\hline $\begin{array}{l}\text { SDRCI (Stephens y Delys, 1973) } \\
\text { (edad preescolar) }\end{array}$ & \multicolumn{2}{|c|}{ De 0,60 a 0,74} & 575 \\
\hline
\end{tabular}

(Original de Rodríguez-Naranjo et al., 1990)

Como puede verse, sin embargo, en algunos casos la estabilidad temporal es superior a la consistencia interna. Así ocurre, por ejemplo, en la escala generalizada de Nowicki y Strickland (Halpin y Ottinger, 1983; Piotrowski y Dunham, 1983; Shriberg, 1974).

A diferencia de ésta, los coeficientes encontrados sobre las dos escalas dirigidas a evaluar el lugar de control sobre áreas específicas, correspondientes a situaciones académicas e interpersonales, son, en bastantes casos, menores que los anteriores; así, en la IAR, los coeficientes obtenidos oscilan de 0,47 a 0,74 (Crandall y col., 1965), y en la CPSI arrojan índices semejantes en las tres subescalas, de 0,68 a 0,70 (Dahlquist y Ottinger, 1983), respectivamente.

Respecto a las escalas dirigidas a edad preescolar, Mischel y col. (1974) calculan, sobre la SPIES, la estabilidad temporal para ambos sexos, ofreciendo indices superiores en el caso de los varones (de 0,55 a 0,70 ) que en el de las niñas, que resultaba en algunos casos no significativa y cuyo coeficiente máximo era de 0,65 . Respecto a las otras dos escalas de preescolar, la PPNS-IE y SDRCI, 
ambas arrojan coeficientes suficientemente elevados para poder afirmar que sus puntuaciones son temporalmente estables (En el primer caso: Nowicki y Duke, 1974. En el segundo: Delys, 1971; Stephens, 1972).

Puede concluirse de estos resultados que la estabilidad temporal de las escalas de lugar de control suele resultar lo suficientemente elevada para justificar, por un lado, un índice generalmente adecuado de fiabilidad de las mismas, y, por otro, para mostrar cómo el lugar de control es un constructo de personalidad con una cierta estabilidad temporal, mayor cuanto más generales sean las escalas. Esto útlimo tiene sentido en la medida en que las expectativas sobre situaciones específicas pueden verse fácilmente afectadas por experiencias particulares, en tanto que cabe predecir que este tipo de experiencias afecten en menor grado a las expectativas sobre un amplio espectro de situaciones y/o tareas.

\section{VALIDEZ DE CONSTRUCTO DE LAS EXPECTATIVAS DE LUGAR DE CONTROL TAL COMO SON EVALUADAS POR LAS PRINCIPALES ESCALAS PARA NIÑOS Y ADOLESCENTES}

\subsection{Relación entre las distintas escalas de lugar de control para niños y adolescentes}

En general, las relaciones de unas escalas con otras resultan bajas en la mayoría de las ocasiones y, en algunos casos, prácticamente nulas. Dado que existen escalas de distinto tipo para evaluar el lugar de control en niños y adolescentes, debería procederse con un cierto orden al realizar las comparaciones entre las mismas. Los autores, sin embargo, no parecen tomar en consideración las características particulares de sus escalas a la hora de comparar sus puntuaciones con las de otras escalas que evalúen el mismo constructo en relación a la misma área de contenido o a las mismas dimensiones.

Además, por otra parte, en aquellos casos en que lo anterior sí es tenido en cuenta, no se encuentran relaciones mayores entre aquellas escalas dirigidas a evaluar un mismo universo de contenido (lugar de control en relación a situaciones académicas, por ejemplo) que entre las escalas de lugar de control generalizado; y éstas tampoco son mayores que las obtenidas sobre aquéllas, dirigidas a evaluarlo en diferentes áreas (como ocurre entre la CPSI y la IAR, dirigidas a situaciones de interacción social y académicas, respectivamente). El mismo tipo de relaciones se han obtenido en aquellas escalas que se diferencian en la naturaleza de las dimensiones evaluadas, encontrándose en general en escasas ocasiones algunos resultados que indiquen algún patrón esperado de relaciones. Como puede verse, los resultados obtenidos (algunos de los cuales se exponen a continuación) son bastante confusos.

Las relaciones entre dos escalas generalizadas, la LCS y la Battle-Rotter, resultan nulas excepto para los grados 6 y 8 , sobre los que sí se obtiene un coeficiente significativo, aunque moderado, $-0,42$ (Battle y Rotter, 1963). Por otra parte, las correlaciones obtenidas entre las dos escalas dirigidas a evaluar el lugar de control sobre asuntos de naturaleza académica - la IAR y la AAA - resultan moderadas, 0,28 a 0,39 (Clifford y Cleary, 1972; Clifford, 1976; Rahe, 1975), y no son superiores que las obtenidas entre la IAR y la CPSI, dirigida esta última a evaluar el lugar de control sobre un área específica diferente, las situaciones de interacción social, con coeficiente de 0,30 sobre la subescala negativa y 0,40 sobre la positiva (Dahlquist y Ottinger, 1983). 
De cualquier forma, las interrelaciones bajas entre las distintas escalas de lugar de control no resultan sorprendentes si tenemos en cuenta que la consistencia interna de las mismas tampoco suele ser tan alta como cabría esperar.

\subsection{Estructura factorial}

Existen numerosos estudios en torno a la estructura factorial de algunas de las escalas de evaluación del constructo sobre poblaciones de sujetos adultos (v. gr. sobre la de Rotter: Collins, 1974; Mirels, 1970; Pérez García, 1984; Strickland y Haley, 1980; Zuckerman y Gerbasi, 1977; etc. Sobre la de Levenson: Fleming y Spooner, 1985; Levenson, 1973, 1974; Levenson y Miller, 1976; Stanley, Hyman y Sharp, 1983), cuyos resultados son interpretados por la mayoría de los autores como prueba de la multidimensionalidad del mismo. Sin embargo, son escasos los estudios factoriales realizados sobre escalas infantiles, limitándose en su mayoría a evaluar la estructura factorial de la CNS-IE de Nowicki y Strickland y la de su versión para niños preescolares, la PPNS-IE.

Mediante la aplicación del procedimiento de análisis de componentes principales y rotación varimax en la CNS-IE, Nowicki (1976) ha encontrado la presencia de tres factores que se mantienen a lo largo de las edades a las que es aplicable la prueba (desde los 8 años hasta la adolescencia). Estos son «Control Personal», «Logro y Madurez» y «Suerte». Los mismos factores son obtenidos por Wolf y col. (1982) y Piotrowsky y Dunham (1983).

Se han obtenido factores diferentes, sin embargo, cuando se han utilizado otras muestras distintas a las de los estudios anteriores. Así ocurre, por ejemplo, en el estudio de Kendall y col. (1978) sobre la estructura factorial de la PPNSIE y de la CNS-IE. Estos últimos autores utilizan para su estudio dos submuestras de niños normales, un grupo de niños emocionalmente perturbados y un último grupo de delincuentes juveniles, obteniendo factores diferentes para cada uno de los grupos. En el caso concreto de la PPNS-IE, los factores que se encuentran en este estudio son diferentes de los obtenidos por Nowicki y Duke (1974), que replicaban en gran medida los encontrados por Nowicki (1976) sobre la CNS-IE en cuanto al contenido de los ítems. En el mismo sentido, Herzberg y col. (1978) tampoco encuentran una estructura factorial estable al utilizar distintas submuestras de sujetos. A partir de estos resultados cabe señalar la existencia de una alta dependencia del número y naturaleza de los factores surgidos en los distintos estudios del tipo de población muestreada.

Por otra parte, puede estar ocurriendo que el método de análisis utilizado sea inadecuado para lo que se pretende comprobar. En definitiva, para la finalidad que se pretende (intentar ofrecer evidencia empírica acerca de los presupuestos teóricos de los autores) parecería más razonable utilizar acercamientos de análisis factorial confirmatorio. Unicamente de esta forma podrán aclararse las relaciones entre las dimensiones teóricas existentes.

\subsection{Validez de constructo de las escalas}

En este apartado se pretende analizar la medida en que las escalas infantiles revisadas anteriormente ven cumplidas sus predicciones teóricas en torno a las relaciones de lo que dicen medir con otras variables psicológicas y, en consecuencia, ofrecer datos en torno a la naturaleza del constructo tal como es evaluado por las mismas. Sin embargo, no todas las escalas de lugar de control 


\section{8}

formulan predicciones teóricas particulares sobre el constructo, limitándose los autores de la mayoría de ellas a calcular las relaciones propuestas con anterioridad.

Entre las escalas más destacadas al nivel de sus predicciones teóricas figura la LCS de Bialer (1961), que tal como se inició anteriormente, ha sido explícitamente construida con el propósito de comprobar la medida en que el lugar de control evoluciona de externo a interno conforme se incrementa la edad de los niños. Esta hipótesis se ve confirmada por el estudio del propio autor de la escala, que obtiene una correlación significativa con la edad cronológica y con la edad mental, 0,37 y 0,56 , respectivamente. También son significativos los resultados en torno a esta variable, cuando se objetiviza a partir del grado de escolarización de los sujetos (Pawlicki, 1974; Penk, 1969) y de su capacidad verbal (Bialer, 1961; Penk, 1969; Riedel y Milgram, 1970).

A partir de estos estudios se ha venido manteniendo la concepción general del lugar de control como una variable que evoluciona con la edad, obteniéndose relaciones significativas en la mayoría de los casos (Milgram y Milgram, 1974; Nowicki y Duke, 1974; Nowicki y Strickland, 1973; Stephens y Delys, 1973).

Igualmente confirmada se ha visto la otra predicción importante realizada sobre la LCS, según la cual los internos deberían mostrar una mayor aceptación de la demora en la gratificación que los externos (Bialer, 1961). Quizá debido a la complicación de su medida, que requiere de un estudio experimental, esta relación se ha visto calculada en muy pocas ocasiones, en las cuales sin embargo sí ha encontrado confirmación empírica (Strickland, 1971, 1972, 1973).

Otra escala que ha ofrecido hallazgos muy importantes para la teoría del lugar de control ha sido la IAR de Crandall y col. (1965). Por un lado, a partir de su naturaleza específica (elaborada para situaciones académicas) y, por otro, a causa de la distinción entre resultados positivos y negativos.

La naturaleza específica de esta escala ha suscitado una gran cantidad de estudios para comprobar sus relaciones con el logro académico, confirmando éstos la capacidad predictiva de la IAR sobre esta variable (Crandall y col., 1965; McGuee y Crandall, 1968; Reimanis, 1973). Los resultados han sido también significativos cuando se ha empleado como medida de logro la ejecución en tareas cognoscitivas (Crandall y Lacey, 1972).

Los hallazgos generales en torno a la relación del logro con el lugar de control han sido positivos (Ludwigsen y Rollins, 1971; Nowicki, 1971; Nowicki y Roundtree, 1971; Nowicki y Strickland, 1973); si bien, éste continúa siendo hoy día un tema controvertido a causa de la cantidad de variables que parecen modularla —edad (Crandall y col., 1965), sexo (Crandall y coll., 1965; Crandall y Lacey, 1972; McGuee y Crandall, 1968; Nowicki y Duke, 1974; Nowicki y Strickland, 1973; Reimanis, 1973; Roberts, 1971), etc.--, sin que todavía se pueda ofrecer un patrón claro de predicciones ajustadas a las características de cada muestra.

La otra aportación importante de Crandall y col., la distinción entre resultados positivos y negativos, no supuso la elaboración de predicciones diferenciadas para su escala. Sin embargo, los resultados obtenidos sobre la misma van en la dirección propuesta. Así, la necesidad de logro (operativizada como la cantidad de tiempo que los niños pasan dedicados a actividades intelectuales durante un período de juego libre) se relaciona con esta escala para $\mathrm{I}+$, pero no para I-. Y en el caso de la edad, esta presenta asociaciones diferentes con la escala para la internalidad positiva y la negativa. En definitiva, cabe destacar cómo esta distinción se ha mantenido en otras escalas elaboradas posteriormen- 


\section{9}

te (la SPIES de Mischel y otros, la escala «Tel Aviv» de Milgram y Milgram, etc.), ofrenciendo en general resultados que apoyan esta distinción.

Por otra parte, la relación que postulan Battle y Rotter (1963) entre las puntuaciones de su escala y el nivel socioeconómico se ha visto confirmada sobre ésta y otras escalas (Battle y Rotter, 1963; Crandall y col., 1965; Franklin, 1963; Gorsuch y col., 1972; Gruen y col., 1974; Nowicki y Strickland, 1973; Powell y Vega, 1972; Strickland, 1971; Wolf y col., 1979, 1982). Y respecto a la otra asociación que esperan se obtenga entre las puntuaciones de su escala y el logro académico, ésta se ha encontrado en algunos de los cursos evaluados y sólo para los varones (Reimanis, 1973). Igualmente controvertidos e incluso contradictorios en algunos casos son los resultados obtenidos en torno a la relación con la inteligencia, que sus autores esperaban fuese nula (Battle y Rotter, 1963; Reimanis, 1973). Como puede apreciarse, la validez de constructo de la escala de Battle y Rotter se confirma en lo concerniente a la clase social, pero como resulta en otros muchos casos, las relaciones calculadas con la inteligencia y el logro académico ofrecen resultados difíciles de interpretar en consonancia con las hipótesis de los autores.

Sí parecen claros, sin embargo, los resultados en torno a la relación con la clase social, la cual se presenta también en la escala de Gruen y col. (1974), a pesar de haber sido elaborada con el propósito de eliminar las diferencias atribuibles a esta variable. Sobre esta escala, sin embargo, sí se ve confirmada por parte de sus autores la esperada ausencia de relación con la inteligencia.

Respecto a la CNS-IE de Nowicki y Strickland, recuérdese que estos autores basan sus predicciones en los estudios realizados sobre escalas anteriores. Así, se ve confirmada tanto la esperada relación con la edad como la asociación con el logro académico (Ludwigsen y Rollins, 1971; Nowicki, 1971; Nowicki y Roundtree, 1971; Nowicki y Strickland, 1973). Igualmente, se confirma su validez discriminante a partir de la ausencia de relación que se encuentra con la deseabilidad social (Nowicki y Strickland, 1973) y con la inteligencia (Nowicki y Roundtree, 1971; Nowicki y Strickland, 1973; Halpin y Ottinger, 1983). Si bien la ausencia de relación con la deseabilidad social no había sido propuesta con anterioridad, sí había sido calculada sobre la LCS de Bialer, resultando igualmente nula sobre una muestra de retrasados mentales, y sobre la IAR de Crandall y col., con resultados más controvertidos.

En este sentido, un aspecto común a la mayoría de las escalas ha sido el considerar la inteligencia y la deseabilidad social como un sesgo en las respuestas, por lo que en algunas de ellas se ha calculado esta relación con el objetivo de comprobar la validez discriminante (Nowicki y Strickland, 1973).

Muy semejante a la escala de Nowicki y Strickland en cuanto a predicciones teóricas es la versión para preescolares de la PPNS-IE de Nowicki y Duke (1974), sobre la que éstas se ven también corroboradas. Así, sus autores encuentran una relación positiva con la edad y el logro académico y negativa con la deseabilidad social.

Respecto a la otra escala para preescolares, la SDRCI de Stephens y Delys (1973), presenta una asociación positiva con la ejecución académica, tal como habían postulado sus autores (Stephens, 1971; Stephens, 1972).

Por último, ya se comentó en el epígrafe correspondiente la ausencia de validez predictiva de la distinción efectuada por Milgram y Milgram (1975) en su escala (TA) entre resultados pasados y futuros. 


\section{0}

\section{DISCUSION}

De la revisión realizada en torno a las escalas de lugar de control en niños y adolescentes cabe extraer algunas conclusiones. En primer lugar, la conveniencia de utilizar las escalas existentes según los propósitos para los que sus autores las crearon, tanto en lo referente a la edad de los sujetos a los que se dirige como a la clase social de la muestra, etc. En la medida en que el lugar de control fluctúa en función de distintas variables psicológicas, será conveniente evaluarlo de forma diferente en función del tipo de variables que caractericen a la muestra sobre la que estemos trabajando. Y en este sentido no parece suficiente con traducir al castellano las escalas de países anglosajones, resultando conveniente bien su adaptación, bien la creación de instrumentos específicos para nuestra población.

Por otra parte, la distinción en función del tipo de resultados, distinguiendo entre el lugar de control para resultados positivos y negativos, de lo cual Crandall y sus colaboradores han sido los pioneros, ha permitido igualmente realizar hallazgos, tanto por estos autores como por otros posteriormente, que corroboran la utilidad predictiva de dicha diferenciación. Además, estos resultados se prestan a investigaciones futuras que, partiendo de los datos ya obtenidos, evalúen la diferente dinámica que opera para asumir la responsabilidad sobre resultados positivos y negativos.

Sin embargo, tal como puede comprobarse por la revisión anterior, la tónica de los estudios sobre el constructo radica en no tener en consideración, en la mayoría de los casos, los resultados obenidos con anterioridad, limitándose a replicar los ofrecidos por la literatura clásica del constructo. El principal problema reside en no tener en cuenta los hallazgos anteriores ni siquiera a la hora de diseñar las escalas de lugar de control.

No obstante esta falta de orden en los estudios, los resultados ofrecidos corroboran la hipótesis inicial de Rotter (1966) en torno a la gran importancia de las expectativas del lugar de control como variable predictora de la conducta. Sin embargo, podemos preguntarnos si las escalas revisadas evalúan todas un mismo constructo o si por el contrario evalúan dimensiones diferentes. $\mathrm{Y}$ nos planteamos esto porque, a pesar de ser todas ellas escalas de «lugar de control», son muchas las diferencias encontradas entre unas y otras. Así, por ejemplo, se comenta anteriormente cómo las dos subescalas (positiva y negativa) de la escala IAR presentan relaciones diferentes con la edad. También aparecen diferencias en los factores muestreados para los polos interno y externo de varias escalas; existen notables diferencias en el grado de controlabilidad que expresan los ítems, tanto internos como externos, etc. En definitiva, todos estos aspectos, junto con la diferente capacidad predictiva que muestran las escalas en función de sus características particulares (este aspecto se acentúa fundamentalmente en relación con el logro académico), vienen a permitirnos concluir, en consonancia con los postulados de Rotter (1975), que el lugar de control es un constructo cuya capacidad predictiva aumenta en función de la especificidad con la que se lo evalúa.

En esta línea, no podemos olvidar tampoco el contexto del que surge el constructo lugar de control, la teoría del Aprendizaje Social, en la que junto a esta variable juega un papel destacado tanto la situación psicológica como el valor del esfuerzo. Si bien la primera ha sido básicamente olvidada al dedicarse la investigación existente en este área de forma casi exclusiva a validar las escalas 
de lugar de control, la capacidad predictiva del constructo sobre la conducta se ve notablemente aumentada en la medida en que se tome en cuenta la situación psicológica, lo que goza de grandes repercusiones en las aplicaciones prácticas del constructo. Respecto a la otra variable importante en el contexto teórico citado, el valor del refuerzo, también con notables repercusiones prácticas, como ya señalamos anteriormente, puede considerarse una aproximación importante en este sentido la distinción realizada por Crandall y col. (1965) entre resultados positivos y negativos.

Para terminar, como decíamos al principio de este apartado, la utilidad predictiva y explicativa del constructo lugar de control depende en gran medida de la adecuación del instrumento de medida empleado para la finalidad con la que se evalúe. En este sentido pretendemos que cuente nuestra aportación, en la medida que pueda ser útil para aquellos que se planteen bien la adaptación de alguna de las escalas ya existentes a nuestra población, bien la creación de algún nuevo instrumento, así como para juzgar acerca de la utilidad del constructo.

\section{Referencias}

Battle, E. S., y Rotter, J. B. (1963). Children's Feelings of Personal Control as Related to Social Class and Ethnic Group. Joumal of Personality, 31, 482-490.

Bialer, I. (1961). Conceptualization of Sucess and Failure in Mentally Retarded and Normal Children. Jounal of Personality, 29, 303.320.

Brewin, C. R., y SHAPIRo, D. A. (1984). Beyond locus of control: Attribution of responsibility for positive and negative ocutcomes. British Journal of Psychology, 75, 43-49.

Clifford, M. M. (1976). A Revised Measure of Locus of Control. Child Study Journal, 6, $85-90$.

Clifford, M. M., y Cleary, T. A. (1972). The Relationships Achievement Accountability. Child Development, 43, 647-655.

Collins, B. (1974). Four Components of the Rotter Internal-External Scale: Belief in a Difficult World, Just a World, a Predictable World, and a Politically Responsive World. Personality and Social Psycbology, 29, 381-391.

Crandall, V. C., y Lacey, B. W. (1972). Children's Perception of I-E Control in IntellectualAcademic Situations and their Embedded Figures Test Performance. Child Development, $43,1.123-1.134$

Dahlquist, L. M., y Ottinger, D. R. (1983). Locus of Control and Peer Status: A Scale for Children's Perception of Social Interaction. Joumal of Personality Assessment, 47 (3), 279-287.

DeLYs, P. (1971). Rationale Method and Validity of the SDRCI-IE Measure for Preschool Children. Comunicación presentada en el American Psychological Association Comentier. Simposio sobre Nuevos Métodos y Proyectos, celebrado en McCandless, Washington D.C.

Fleming, J. S., y SPOONER, P. S. (1985). Five Factor Scales for Internal-External Control and their Relations to Measures of Adjustment. Journal of Clinical Psychology, 41 (4), 512-517.

FRANKLIN, R. D. (1963). Youth's Expectancies about Internal versus External Control of Reinforcement Related to $N$ Variables. Tesis Doctoral. Universidad de Purdue.

GrLmor, T. M. (1978). Locus of Control as a Mediator of Adaptative Behavior in Children and Adolescents. Canadian Psychological Review, 19 (1).

Godoy, A.; Rodríguez.Naranjo, C.; Esteve, R., y Silva, F. (1989). Escalas de Lugar de Control en Situaciones Académicas (ELC-A) y en Situaciones Interpersonales (ELC-I) para Niños y Adolescentes. Evaluación Psicológica, 5 (3), 273-322.

Gozali, J., y Bialer, I. (1968). Children's Locus of Control Scale: independence from Response set Bias Among Retardates. American Joumal of Mental Deficiency, 72, 622-625.

Gregory, W. L. (1978). Locus of Control for Positive and Negative Outcomes. Joumal of Personality and Social Psycbology, 36, 840-849.

Halpin, B. M., y OTtinger, D. R. (1983). Children's Locus of Control Scales: A Reappraisal of Reliability Characteristics. Child Development, 54, 484-487. 
Herzberger, S. H., et al. (1978). Preschool and Primary Locus of Control Scale: Is it Ready for Use? Developmental Psychology, 3, 320-324.

JAMES, W. H. (1957). Intemal versus Extemal Control of Reinfoncement as a Basic Variable in Learning Theory. Tesis Doctoral. Universidad de Ohio.

JoE, V. C. (1971). Review of Internal-External Control Construct as Personality Variable. Psychological Reports, 28, 619-640.

Julian, J. W., y Katz, S. B. (1968). Internal versus External Control and the Value of Reinforcement. Journal of Personality and Social Psychology, 9, 363-368.

KenDALL, P. C., et al. (1978). Variations in a Construct: Cuantitative and Cualitative Differences in Children's Locus of Control. Jourmal of Consulting and Clinical Psychology, 46, $590-592$.

Levenson, H. (1974). Activism and Poweful Others: Distinctions within the Concept of Internal-External Control. Joumal of Personality Assesment, 38 (4), 377-383.

Levenson, H. (1973). Perceived Parental Antecedent of Internal, Powerful Others, and Chance Locus of Control Orientations. Developmental Psychology, 9, 260-265.

Lewin, R. (1935). A Dynamic Theory of Personality.: N. Y. McGearo-Hill.

LUDWIGSEN, K., y Roluins, H. (1971). Recongnition of Random Forms as a Function of We. Perceived Locus of Control, and Socio-economic Level. Póster presentado al Encuentro Anual de la Asociación Psicológica del Suroeste, celebrado en Miame Beach, Florida.

McGueE, P. E., y CrandaLL, V. C. (1968). Belief in Internal-External Control of Reinforcements and Academic Performance. Child Development, 39, 91-102.

Milgram, N. A. (1972). Cognitive and Attitudinal Motivational Factor in the Performance of Retarded Persons (Grant N. ${ }^{\circ}$ RD-2569-R). Universidad de Temple. Servicio Social y de Rehabilitación.

Milgram, N. A., y Milgram, R. M. (1975). Dimensions of Locus of Control in Children. Psychologycal Reports, 37, 523-538.

Milier, M. B. (1960). Reliability of the Bialer-Cromwell Scale in a Mentally Retarded Group. Abstracts of Peabody Studies in Mental Retardation, 1 (73).

Mirels, H. L. (1970). Dimensions of Internal versus External Control. Joumal of Consulting and Clinical Psychology, 34, 226-228.

Nowicki, S. (1971). Acbievement a Popularity as Related to Locus of Control Across Different Age Groups. Tesis Doctoral. Universidad de Emory.

NowICKI, S. (1976). Factor Structure of Locus of Control in Children. Joumal of Genetic Psychology, 129, 13-17.

NowICKI, S., y DuKE, M. P. (1974). A Pre-School and Primary Internal-External Control Scale. Developmental Psychology, 10, 874-880.

Nowicki, S., y StickLand, B. R. (1973). A Locus of Control Scale for Children. Joumal of Consulting and Clinical Psychologic, 4, 148-155.

PawLICKI, R. E. (1974). Locus of Control and the Effectiveness of Social Reinforcers. Journal of Genetic Psychology, 125, 153-158.

Pelechano, V., y Báguena, M. J. (1983). El Cuestionario Lucad: Locus de Control para Niños y Adolescentes. Análisis y Modificación de Conducta, 9 (22), 349-392.

PENK, W. E. (1969). Age Change and Correlaties of Internal-External Locus of Control Scale. Psychological Reports, 25, 856-861.

Phares, E. J. (1957). Expectancy Change in Skill and Chance Situations. Joumal of Abnormal and Social Psychology, 54, 339-342.

Phares, E. J., y Lamiell, J. T. (1974). Relationship of Internal-External Control of Defensive Preferences. Joumal of Consulting and Clinical Psychology, 42 (6), 872-878.

Piotrowski, C. y Dunham, F. Y. (1983). Stability of Factor Structure in Fifth Grades on the Children's Nowicki-Strickland Internal-External Control Scale. Joumal of Psycbology, 115, 13-16.

PoweLL, A., y VEGA (1972). Correlates of Adult Locus of Control. Psychological Reports, 30, 455.460.

RaHE, D. F. (1975). Locus of Control and Preferences of Children for Skill versus Chance Tasks. Tesis doctoral. Universidad de Iowa.

Reimanis, G. (1973), School Performance, Intelligence, and Locus of Reinforcement Control Scales. Psychology in the Schools, 10, 207-211.

Riedel, W. W., y Milgram, N. A. (1970). Level for Aspiration Locus of Control and NAchievement in Retatardated and Normal Children. Psycbological Reports, 27, 551-557.

RoBERTs (1971). The Self Esteem of Disadvantaged Third and Seventh Graders. Tesis doctoral. Universidad de Emory.

Rorrer, J. B. (1954). Social Leaming and Clinical Psychology. Nueva Jersey: Englewood Cliffs.

Rotrer, J. B. (1975). Some Problems and Misconceptions oriented to the Construct of Internal versus External Control of Reinforcement. Joumal of Consulting and Clinical Psycho$\log y, 43,56-67$

RoTTER, J. B. (1966). Generalized Expectancies for Internal versus External Control of Reinforcement. Psychological Monographs, 80 (1), 1-28. 


\section{3}

Seeman, M. (1963). Alienation and Social Learning in a Reformatory. American Joumal of Sociology, 270-284.

Shriberg. (1974). Descriptive Statistics for Two Children's Social Desirability Scales, General and Test Anxiety and Locus of Control in Elementary School Children. Psychology Reports, 34, 863-870.

Skinner, E. A., y Chapman, N. (1987). Resolution of a Developmental Paradox: How Can Perceived Internality Increase, Decrease, and Remain the Same Across Middle Childhood? Developmental Psychology, 23, 44-48.

Stephens, M. W., y Delys, P. (1973). A Locus of Control Measure for Pre-school Children. Developmental Psychology, 9, 55-65.

StePHENS, N. W. (1972). Dimensions of Locus of Control: Impact of Early Educational Experiences. Proceedings of the 80 Annual Convention of the American Psychological Association, 7 (pp. 137-138).

StepHENS, N. W. (1971). Evaluation of Locus of Control Measures for Follow Hirongh Evaluation. Universidad de Purdue.

StrickLAND, B. R. (1971). Aspiration Responses Among Negro and White Adolescents. Journal of Personality and Social Psychology, 31, 410-422.

StRICKLAND, B. R. (1972). Locus of Control and Competence in Children. Póster presentado al Encuentro Anual de la Asociación Psicológica Americana, celebrado en Honolulú, Hawai.

STRICKLAND, B. R. (1973). Locus of Control: Where Have We Being and Were are We Going? Póster presentado al Encuentro Anual de la Asociación Psicológica Americana, celebrado en Montreal, Quelsec.

StrickLAND, B. R., y HaLeY, W. E. (1980). Sex Differences on the Rotter Internal-External Scale. Journal of Personality and Social Psychology, 39, 930-939.

WeINER, B. (1980). A Cognitive (Attribution) - Emotion- Action Model of Motivated Behavior: An Analysis of Judgments of Help-Giving. Joumal of Personality and Social Psychology, 39 (2), 186-200.

WeInER, B. (1979). A Theory of Motivation for some Classroom experiens. Joumal of Educational Psychology, 71 (3-25).

WoLf, T., et al. (1982). Factor Analytic Studie of the Children's Nowicki-Strickland Locus of Control Scale. Educational and Psychological Measurement. 42, 333-337.

Zuckerman, M., y Gerbasi, K. C. (1977). Dimensions of the Internal versus External Scale and the Relationship to Other Personality Measure. Educational of Psychology Measurement, 37, 157-175. 